\title{
Anabases
}

ANABASES Traditions et réceptions de l'Antiquité

17 | 2013

Varia

\section{Pirro Ligorio (1512-1583) et les véhicules antiques}

\section{Ginette Vagenheim}

\section{OpenEdition}

Journals

Édition électronique

URL : http://journals.openedition.org/anabases/4129

DOI : 10.4000/anabases.4129

ISSN : 2256-9421

\section{Éditeur}

E.R.A.S.M.E.

\section{Édition imprimée}

Date de publication : 1 mars 2013

Pagination : 85-103

ISSN : 1774-4296

\section{Référence électronique}

Ginette Vagenheim, «Pirro Ligorio (1512-1583) et les véhicules antiques », Anabases [En ligne], 17।

2013, mis en ligne le 01 avril 2016, consulté le 20 octobre 2019. URL : http://journals.openedition.org/ anabases/4129; DOI : 10.4000/anabases.4129 
Anabases 17 (2013), p. 85-103.

\section{Pirro Ligorio (1512-1583) et les véhicules antiques}

GinetTe VAGENheim

EN 1671, JoHANNES SCHEFFerus (SCHEFFer), professeur d'éloquence à l'université d'Uppsala (1621-1679), publie à Francfort, chez Zunner, un volumineux traité en deux livres sur les moyens de locomotion chez les Anciens : De re vehiculari veterum ("Les véhicules chez les Anciens »). En appendice à l'ouvrage, l'érudit strasbourgeois publie une traduction latine commentée d'un mémoire sur le même sujet, tiré de l'œuvre du célèbre antiquaire napolitain Pirro Ligorio (1512-1583), l'une des figures les plus fascinantes de la Renaissance par sa personnalité et par l'œuvre immense qu'il nous a léguée dans les domaines les plus divers ${ }^{1}$.

Ligorio était à la fois peintre, dessinateur, architecte, ingénieur et " antiquaire ", c'est-à-dire un autodidacte qui s'est consacré à l'étude des différents aspects du monde antique, et en particulier du monde romain, en se fondant sur toutes les sources à sa disposition. Arrivé de Naples vers 1545, il se consacre aussitôt à l'étude de la Rome antique, par ses fouilles et ses investigations des monuments et des autres sources, notamment épigraphiques et numismatiques. Les résultats de ses recherches furent ensuite recueillis dans une œuvre en plusieurs volumes, restée manuscrite et intitulée Les Antiquités Romaines (Antichità Romane). Celle-ci forme une encyclopédie monumentale du monde antique illustrée de dessins, composée de vingt-cinq livres sur les monnaies grecques et romaines, cinq livres sur les inscriptions, trois livres sur les bustes

1 Qu'il me soit permis de dédier cet article à Mirella Ferrari en signe de mon affectueuse reconnaissance qui dure depuis plus de vingt-cinq ans. Je remercie cordialement AnneHélène Klinger-Dollé pour sa lecture minutieuse de mon article et pour les nombreuses améliorations consécutives; les erreurs qui subsistent me sont entièrement imputables. 
d'hommes illustres, et de dessins de statuettes, gemmes, sarcophages, monuments funéraires, arcs, plans de temples et d'autres monuments antiques présents à Rome et dans le reste de l'Italie. À la même date, Ligorio entre au service du cardinal Hippolyte II d'Este (1509-1572) pour une durée de six ans et commence les fouilles à la Villa d'Hadrien ainsi que la construction de la Villa d'Este à Tivoli ; à cette époque, il s'occupe aussi de la décoration d'autres villas romaines dont il orne les jardins de statues antiques et d'autres monuments découverts au cours de ses fouilles. En 1549, Ligorio entre au Vatican et publie également le résultat de ses recherches archéologiques, notamment ses cartes topographiques de la Rome antique, un traité sur les théâtres et les cirques, une reconstitution des thermes de Dioclétien et Maximien, celle de la volière antique de l'auteur latin Varron (Aviarium) et ses illustrations des fables d'Ésope. En 1555, l'antiquaire entre au service du pape Paul IV et devient architecte au Vatican. Il y fait de nombreux travaux, parmi lesquels la nouvelle cour du Belvédère et la réalisation du magnifique "Casino " de Pie IV dans les jardins. Cependant, sa fonction la plus importante est celle d'architecte de Saint-Pierre, qu'il occupe à la place de MichelAnge. On lui doit aussi le projet de la tombe de Paul IV conservé à Santa Maria sopra Minerva. En 1567, sous Pie V, Ligorio quitte Rome pour Ferrare, après avoir vendu au cardinal Alexandre Farnèse, sous la pression d'érudits comme Fulvio Orsini et Onofrio Panvinio, les dix volumes de ses Antiquités Romaines conservés aujourd'hui à la Bibliothèque nationale de Naples; sur le chemin, il fait une nouvelle halte à Tivoli où il achève ses travaux à la Villa d'Hadrien et à la Villa d'Este. Il se met au service du neveu d'Hyppolite II, Alphonse II de Ferrare, comme antiquaire de cour pour l'organisation des collections d'antiquités du duc, et comme architecte, notamment pour reconstruire le château d'Este après le tremblement de terre de 1570 dont il nous a transmis le récit circonstancié ${ }^{2}$. Il participe aussi à la scénographie des fameux tournois de Ferrare (cavallerie ferraresi) mais surtout rédige une nouvelle version en trente volume de ses Antiquités Romaines conservés aujourd'hui aux archives d'État de Turin ${ }^{3}$.

Au moment de publier le traité de Ligorio sur les véhicules anciens (Pyrrhi Ligorii Neapolitani de vehiculis antiquorum diatriba: "Traité de Pirro Ligorio napolitain sur les véhicules des anciens »), Scheffer précise que le texte est inédit et conservé dans les fameux Libri delle Antichità romane de Ligorio, et plus exactement dans les livres consacrés aux familles romaines : Excerpta ex libris eius de familiis romanis ("Extraits de ses livres sur les familles romaines " $)^{4}$. Scheffer nous apprend, dans sa lettre au lecteur,

2 Le traité de Ligorio qui présente la première maison antisismique, a été édité par E. Guidoboni, Libro di diversi terremoti, Roma, De Lucca, 2006.

3 Pour plus d'informations sur la biographie de Ligorio : G.VAgenheIm, LIGORIO, Pirro, in P.F. Grendler (éd.), Encyclopedia of the Renaissance, III, New York, Charles Scribner's Sons, 1999, p. 425-427.

4 J. Schefferus, De re vehiculari veterum libri duo. Accedit Pyrrhi Ligorii v.(iri) c.(larissimi) de vehiculis fragmenta numquam ante publicatum ex bibliotheca sereniss(imae) reg(inae) Christina. Cum ejusdem I(ohannis) Schefferi arg.(entoratensis) annotationibus. I-II., 
qu'il a reçu ce texte de son collègue, Andreas Nordenhielm, qui le lui avait rapporté de Rome, où il l'avait copié sur l'exemplaire des manuscrits ligoriens conservés dans la bibliothèque de la Reine Christine de Suède ${ }^{5}$. C'est le caractère inédit du sujet et la réputation de Ligorio qui avaient poussé Scheffer à publier le traité ; mais il s'était vite rendu compte des limites de l'antiquaire, et avait alors décidé de se pencher à son tour sur le sujet ${ }^{6}$.

Au fil de la lecture des premiers chapitres du traité ligorien et des commentaires de Scheffer aux différentes catégories de véhicules, nous verrons ce que les critiques de Scheffer nous révèlent sur la méthode de travail de Ligorio et chercherons à les comprendre en fonction de la personnalité et de la formation de l'antiquaire et dans le contexte culturel de la rédaction des Antichità romane; nous nous interrogerons sur les raisons de la fascination de Scheffer pour le traité ligorien et les raisons qui l'ont poussé à se lancer à son tour dans l'étude des moyens de locomotion chez les Anciens; nous verrons enfin le profit que certains contemporains de Ligorio et de Scheffer ont su tirer des Antichità romane, qui fait encore aujourd'hui de cette œuvre une source précieuse pour l'étude de l'érudition classique à la fin de la Renaissance

Dans son commentaire au traité ligorien, Scheffer fait montre d'une grande réserve à propos de la doctrine et de la méthode de l'antiquaire, même s'il lui arrive quelquefois d'être d'accord avec Ligorio ; c'est le cas, par exemple, au premier paragraphe, à propos du terme désignant le moyen de locomotion : Premieramente porremo le cose in generale. Perché tutti $i$ carri sono detti vehiculi, sotto qualsivoglia altro nome, questo fu il primo che si diede a tutti i carri. Scheffer admet qu' "il est correct de dire vehicula "pour carrus" car le carrus est une espèce de vehiculum et non une catégorie ${ }^{7}$ "; mais avant la fin du paragraphe, il récuse la définition ligorienne du plaustrum: Sotto nome di plaustro, sono molti carri altrimente [sic] detti, secondo è piaciuto a scrittori chiamarli et secondo le nationi che gli hanno usati. Selon Scheffer, il ne s'agit, en aucun cas, cette fois, d'un nom commun qui serait, comme dit Ligorio, l'équivalent du vehiculum, et que les auteurs utiliseraient selon leur bon vouloir ${ }^{8}$; selon lui, le terme vient de l'ad-

Franconfurti, ex officina zunneriana, 1671. Sur la vie de Scheffer : R.Hoche, Scheffer, Johannes Gerhard, in Allgemeine Deutsche Biographie, Band 30, Leipzig, Duncker und Humblot, 1890, p. 680.

5 Les manuscrits ligoriens de la Reine Christine sont aujourd'hui conservés à la Bibliothèque Apostolique Vaticane dans le fonds des Ottoboniani latini, sub nomine. À propos de la polémique autour des manuscrits ligoriens, on se reportera à A. GRISERI, "Quei "Benedetti Ligorii" alla corte di Cristina di Francia ", in C.volPI (a cura di), Il libro dei disegni di Pirro Ligorio all'archivio di stato di Torino, Roma, edizioni dell'Elefante, 1994, p. 31-43.

$6 \quad$ L'appendice I présente le sommaire de l'œuvre de Scheffer.

7 Rectius vehicula dicerentur; nam carrus species vehiculi, non genus. ("Il est plus correct de les appeler véhicules; en effet, carrus désigne une espèce, non une catégorie. ")

8 Putat, plaustrum quoque generale nomen esse. Ac sic saepe in sequentibus. Sed hoc falsum. ("Il pense que plaustrum aussi est un nom commun et il le répète souvent dans la suite; mais c'est faux. ") 
jectif plautus et désigne donc un véhicule large et plat sans aucune structure ni siège (Nobis plaustrum dici a plauto videtur, quia vehiculum fuit latum, id est non in ambitum structum tabulis, aut cum sella superne, verum planum: "Il me semble que le plaustrum vient de plautus, parce que ce type de véhicule était large, c'est-à-dire sans structure de bois, ni siège dessus mais tout à fait plat ${ }^{9}$.")

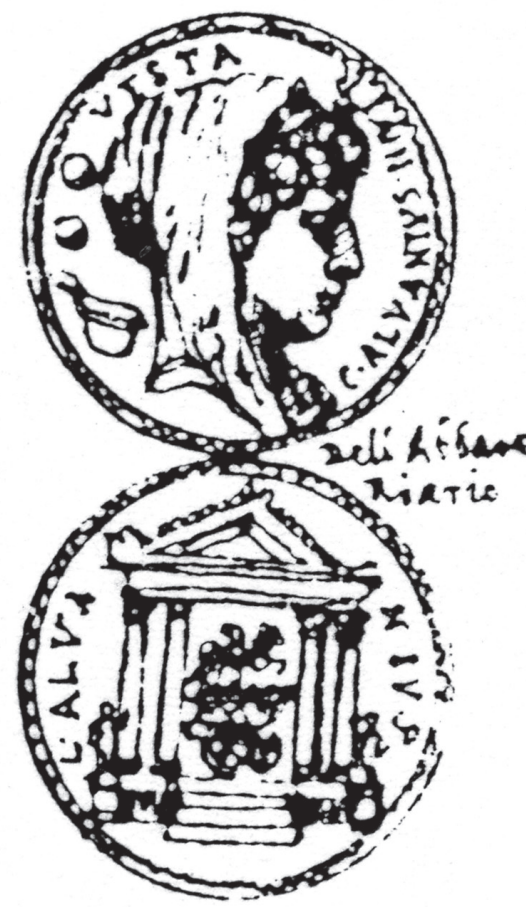

Fig. 1. Torino, Archivio di Stato, Ja.II.6, f.89r.

Au début du traité, Ligorio nous apprend que c'est l'étude des monnaies et leur riche iconographie qui ont motivé son entreprise, en particulier les monnaies émises à l'occasion de victoires militaires ou pour des motifs religieux molte volte, "molte " cose nei carri si veggono poste nei danari [...], o per segno di vittoria o di cose sagre. Pour mieux comprendre la place de ce traité dans son Livre sur les familles romaines, il faut retourner au manuscrit original, conservé aujourd'hui à Turin ${ }^{10}$, sur lequel avait été réalisée la copie conservée dans la bibliothèque de Christine de Suède. Le traité commence précisément un chapitre plus haut, sous le titre suivant : Dell'Alvanii et della sorte de carri antichi ; c'est donc l'exposé sur la famille des Albanii, dont le nom apparaît également sur les monnaies, qui a donné à Ligorio l'occasion de traiter des différentes sortes de chars anciens. En effet, outre le nom de la famille Albanius, Ligorio prétend avoir vu la représentation d'un char dessiné au milieu d'un temple sur le revers d'une monnaie de bronze, conservée dans la collection de l'abate di Riario ; Ligorio en dessine les deux faces en marge du premier paragraphe (fig. 1) : In questo danaro dunque di bronzo, che è nel studio dell'abate Riario, vediamo due Alvanii nominati Caio e Lucio Caio Alvanio dalla "parte " dell'effigie di Vesta, dea del regimento; sotto nome di triumviro della zecca, dallato del tempio, è il detto Iove Alvanio ove dentro il tempio è il carro vittoriato, come cosa di rispetto religioso. Onde io mi penso che sia memoria del carpento, o vehiculo o carro, come lo vogliamo chiamare,

9 SCHEFFERUS, De re vehiculari, p. 244.

10 Torino, Archivio di Stato, Ja.II.6 : Libro XIII di Pyrrho Ligorio, patritio napolitano, et cittadino romano, dell'antichità, nel quale si contiene delle più chiare fameglie romane, con la particolar dichiaratione delle cose fatte et applicate ai soggetti scolpiti nelle loro medaglie, ff. $89 \mathrm{r}-94 \mathrm{v}$. 
di quel Alvanio, che per animo religioso, dispreggò l'interessi suoi istessi per fare che la religione havesse il suo honore. Laonde Caio Alvanio nel magistrato della zecca "fu " ricordevole dell'antico Lucio che fu cagione che il carro ancho havesse luogo nelle sacre cose de Romani.

Ligorio commence par décrire l'image de Vesta sur l'avers de la monnaie ; la déesse, qualifiée de dea del regimento ${ }^{11}$, est voilée et représentée de profil, la tête tournée vers la droite ; derrière sa tête apparaissent des instruments de sacrifice, sans doute deux patères et un vase, surmontés de l'inscription VESTA ; face à son visage, on lit l'inscription à C.(aius) Alvanius IIIvir dont Ligorio précise qu'il était triumviro dela zecca, c'est-à-dire l'équivalent du triumvir monetalis; le revers montre un temple avec un char triomphal en son centre, le carro vittoriato, qui serait un signe de respect (come cosa di rispetto religioso) et sur le côté du temple, une statue de Jupiter Alvanius.

À ce point de l'exposé, Ligorio fait une digression pour expliquer la présence du char sur la monnaie ; d'après lui, il s'agirait de celui que le triumvir Caius Alvanius aurait fait représenter sur la monnaie en souvenir de son ancêtre Lucius dont l'action a permis au carrus d'entrer dans le domaine religieux. Ligorio traduit ensuite, presque mot pour mot, l'histoire que raconte Valère-Maxime au début de ses Actions et paroles Mémorables (I,1), pour illustrer le fait que même de simples particuliers étaient capables de placer la religion au premier rang: Come scrive Valerio Maximo nel libro primo al capo primo, dove egli parla di Lucio Furio Bibaculo et di esso Alvanio, huomini religiosi dela stirpe plebea, il quale coll'osservata religione fece una cosa degna di laude et nobilitò la casa. Costui dunque, tornando dala villa sul carpento, cio è carro con la moglie et figliuoli, in quel istante che i Galli Senoni havevano presa Roma, incontratosi coi sacerdoti di Romolo appellati quirinali e colle vergini Vestali con tutte le loro reliquie e cose sacre degli iddij, che havevano in gran custodia, secondo il rito dei Gentili, che già fuggevano et havevano passato il ponte Sublicio nella montata del colle Ianiculo, si fermò, et quantunque egli fusse plebeo, tosto che conobbe il fatto, levato la moglie et i figliuoli dal carro, il quale come a quello che più le premeva e stringeva il zelo della publica religione, che la privata affettione dela moglie et dei figliuoli ivi accomodò sopra le vergini et con tutte quelle cose sacre l'accompagnò infine alla fortezza dela città di Caere di Toscana discosto ventiquattro miglia da Roma, dove con grandissimo honore quivi ricevute et honorate et custodite con sollenne caerimonie. Onde doppo la cacciata di Galli da Furio Camillo ritornate nella patria, fu fatta memoria di quella religiosa humanità et di Alvanio et dei Caeretani et in testimonio chiamarono le sacre sollenità da Caere, Caerimonia, tutto per reverenza che fu havuta alle cose degli iddij. Perlo che anchora quel carro così vile et così obietto, havendo in tale necessità servito a portare cose stimate santissime et divine, fu reputato che non solo pareggiasse, ma avanzasse ancho la gloria di qualunque altro splendidissimo et ornatissimo carro triomphale.

11 Le sens de ce qualificatif est lié au verbe reggere et indique sans doute une capacité à définir des règles de comportement voire de gouvernance. 
Ligorio conclut en précisant que ce char à deux roues, appelé tout à tour carrus, vehiculus, carpentum ou plaustrum, se trouvait dans le temple de Vulcain ou de Jupiter et qu'il fut utilisé, avant l'arrivée des chars dorés, dans les premiers triomphes, c'està-dire per segno di vittoria, comme il l'avait indiqué au début de son traité : Alcuni questo carro chiamano vehiculo, altri carpento, o plaustro, o come si voglia sia, si vede in questa medaglia con due ruote, et deve essere posto nel tempio di Volcano, o pure di Iove et vogliono che quei primi triomphanti con esso triumphavano : nanzi che venissero i carri aurati et ricchi.

Après la définition générale du vehiculum, Ligorio va consacrer un chapitre plus ou moins long à chacun des vingt-neuf types de véhicules qu'il a répertoriés dans les sources grecques et latines (appendice II) : I Latini hanno usato dunque di dire vehiculum, rheda, essedam, cisium, carpentum, currus, pilentum, arcina, bastarna, benna, biga, triga, quadriga, birotum, burdones, camulum, cantherion, carruca, combatus, covinus, octophorum, betoritum, sarracum, staticulum, thensa, traha, vectabulum et veha. I Greci

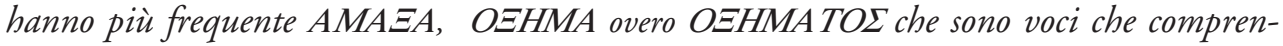
dono molti carri, come fa il nome vehicolo et carpento et curro pressi Latini.

Le premier paragraphe relatif au plaustrum commence, comme d'habitude, par l'énumération des différentes formes linguistiques du terme étudié, en précisant son équivalent en grec, avant d'en rechercher l'étymologie, que Ligorio trouve, dans ce cas, dans la Souda; il cite ensuite les sources littéraires qui nous transmettent les termes plaustrum ou amaxa: Plaustrum, i Greci Amaxa et $A M A \Xi A$ lo dicono et tanto i poeti come gli historici di cui parla Aulu Gellio ${ }^{12}$ del plaustro posto in cielo tra le stelle et ne parlano Arato et Hygino.

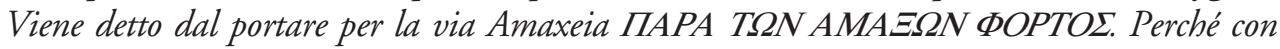
esse si conduce per strada larga et aperta. Plaustro chiamano la quadriga di Erichtonio come altri lo dicono vehiculo, il quale era con due ruote tirato da quattro cavalli, et plaustro et vehiculo et amaxa chiamano il carro di Syfello, tiranno di Corintho, come quel del Sole et quel di Marte, et vi aggiungono volucris, perché hanno i cavalli ale. Plaustro, vehiculo et amaxa ancora furono detti carri di Dario Re quel di Alexandro Magno, quel di Laio, re di Thebe di Beotia, perché tutti suonano una cosa istessa.

Scheffer s'irrite de l'imprécision de Ligorio qui ne cite pas ses sources quand il affirme que le quadrige d'Érichthon était appelé plaustrum: Hoc non lectum mihi ("je ne l'ai pas lu »); ni quand il rapporte que celui du tyran Cypselos, et non Syfello comme dit Ligorio, était désigné par le terme d'amaxa ${ }^{13}$. Scheffer, quant à lui, semble vouloir

12 Aulu-Gelle, Nuits attiques, 2, 21 : Super eo sidere, quod Graeci $\alpha \mu \alpha \xi \alpha v$, nos «septentriones» uocamus; ac de utriusque nocabuli ratione et origine. ("Sur cette constellation que les Grecs nomment $\dddot{\alpha} \mu \alpha \xi \alpha v$ (le Chariot), et que les Latins appellent septentriones. Explication de l'étymologie de ces deux mots. »)

13 Vellem itaque ut auctorem idoneum nominasset. Syfeli videtur Cipselum intelligere sed de curru eius talis nil reperio apud alios. ("C'est pourquoi je souhaiterais qu'il nomme précisément sa source. Par Syfelus, il semble qu'il faille comprendre Cipselus mais je ne trouve rien de tel sur ce char chez les autres auteurs. ») 
mettre un point d'honneur à citer le plus grand nombre de sources avec précision et c'est ainsi qu'il nous fournit une liste impressionnante d'auteurs ayant cité le plaustrum, tant anciens que chrétiens, byzantins, humanistes et contemporains ${ }^{14}$ : Scheffer ne néglige pas non plus le témoignage des monnaies et présente enfin le dessin du plaustrum tel qu'il l'a reconstitué à partir de l'examen des sources consultées (fig. 2).

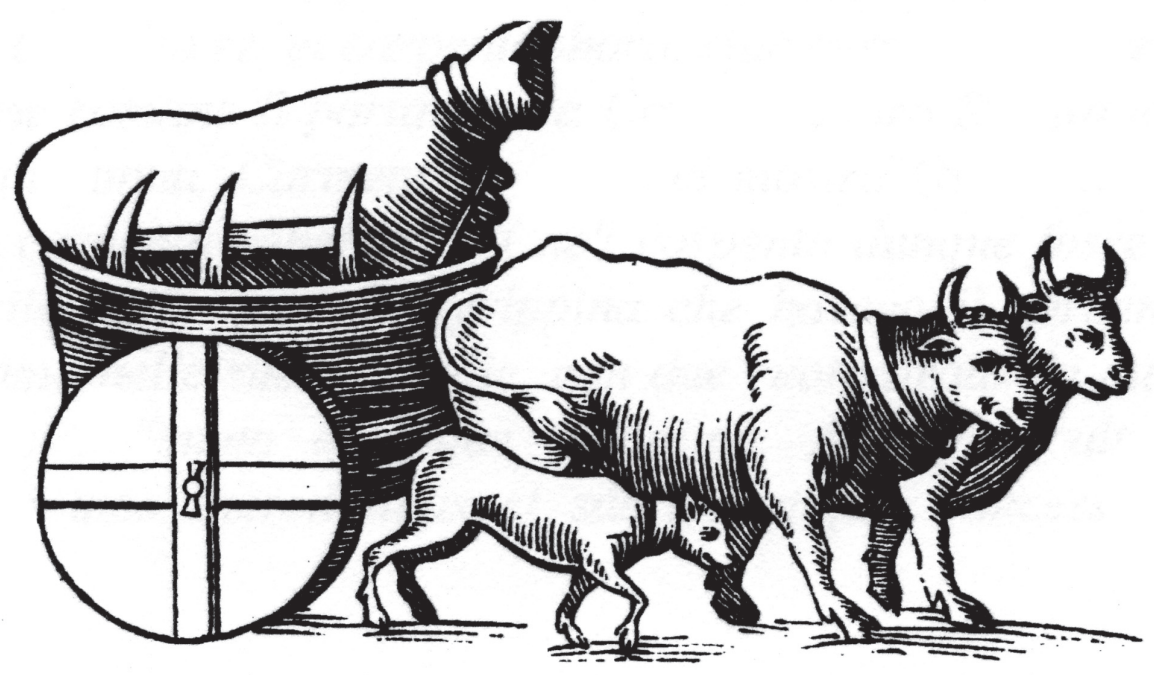

Fig. 2. Plaustrum, in J. Scheffer, De re vehiculari, p. 246.

Le deuxième chapitre du traité ligorien est consacré au carpentum : Carpento fu il primo nome, detto per mutatione di lettere; perché è come dire quasi carmentum, per essere stato usato da Carmenta, madre di Evandro re, et carpentophoro, ciò̀ curro da portare frutti, nelle decime, nei sacrificii, come le donne romane li portavano a Carmenta loro Sybilla et agli altri iddii. Fu detto veramente da essa gran donna, Carmentum, come ci mostra Ovidio nel primo dei Fasti : "Nam prius Ausonias matres carpenta vehebant. " Che'l carpento dunque fosse delle gran donne, celo accenna Cornelio Tacito nelle cose di Agrippina che haveva il carpento fastigiato et bello et ornato, come lo veggiamo nelle sue medaglie, con due ruote tirato da due mule o da due cavalli; onde esso autore dice: "Suum

14 Aristophane, Arrien, Appien, Aratos, Eusthate, Hippocrate, Hérodote, Homère, Lucien, Philoxène, Plutarque, Pollux, Quintus de Smyrne, Spartien, Strabon, Ulpien, Xénophon ; parmi les auteurs latins, Aulu-Gelle, Ammien Marcellin, Caton, Cicéron, Columelle, Festus, Florus, Frontin, Gordien, Horace, Hygin, Isidore de Séville, Justin, Plaute, Pline, Probus, Quinte-Curce, Quintilien, Suétone, Tacite, Tite-Live, Valère-Maxime, Varron, Végète, Virgile ; sont également cités Servius, Maximien, Gualtherus, Eustathe, Cinnamus, Lorenzo Valla, Érasme, pour sa traduction de Lucien, Isaac Vossius, Joseph Juste Scaliger, Matthias Bernegger ainsi que le scholiastes vetus Aristophanis in nubibus. 
quamque fastigium, Agrippina altius extollere, carpento Capitolium ingredi, qui mos sacerdotibus et sacris antiquis concessus, venerationem augebat foeminae. "

Cette fois, Ligorio cite ses sources avec précision en commençant par le vers 619 du premier livre des Fastes: Nam prius Ausonias matres carpenta vehebant ("En effet, les matrones d'Ausonie se déplaçaient en char »); mais l'on découvre qu'il se fonde, sans le citer, sur le contenu du vers suivant pour expliquer l'origine présumée du terme carpentum : Haec quoque ab Euandri dicta parente reo (v. 620 : "Du nom, je crois, de la mère d'Évandre »). Si Scheffer ne conteste pas l'interprétation ligorienne des vers d'Ovide il réfute en revanche, vigoureusement, l'existence du terme carpentophorus « qui n'est attesté nulle part pour désigner un véhicule ${ }^{15}$ »; il n'accepte pas non plus le sens que Ligorio donne à ce terme et qui est le résultat d'une interprétation erronée du terme carpophoros; cet élément prouve, selon Scheffer, que Ligorio ignorait le grec : plane imperitia linguae graecae obstitit Ligorio. ("L'ignorance de la langue grecque fut un obstacle pour Ligorio. ") Quant aux dîmes que celui-ci évoque, l'érudit précise que les Romains n'en connaissaient pas du tout l'usage : decimae, quas hic memorat, Romanis penitus fuerunt ignotae. ("Les dîmes qu'il évoque ici étaient totalement inconnues chez les Romains. »)

Le second auteur cité par Ligorio est Tacite, dans le passage des Annales où l'auteur latin évoque le renforcement du prestige d'Agrippine, qui se manifeste par sa montée au Capitole sur un carpentum (XII, 43) ${ }^{16}$. Ligorio qualifie le véhicule d'Agrippine de carpento fastigiato, ce qui donne à Scheffer une nouvelle occasion de railler Ligorio et de suggérer son ignorance des langues classiques; en effet, après avoir reproduit la phrase correcte de Tacite (suum quoque fastigium Agrippinae altius extollere) ${ }^{17}$, l'érudit qualifie l'explication de Ligorio de totalement inepte (Quod est prorsus ineptum: "Ce qui est totalement inepte »). Est-ce Ligorio le responsable de l'interprétation "inepte " du terme fastigium qu'il a traduit au sens propre, en en faisant le " toit " de la voiture d'Agrippine, ou cette interprétation lui vient-elle d'une tierce personne, comme semble le suggérer ailleurs Scheffer, quand il suppose que Ligorio ne consultait pas directement les sources mais se servait de résumés confectionnés par autrui et qu'il ne les comprenait pas toujours parfaitement (plane suspicor, scriptores ipsos non consuluisse Pyrrhum, verum habuisse nescio a quo confecta quaedam excerpta, ipsi quoque non intellecta satis: "Je soupçonne fortement Ligorio de ne pas avoir consulté directement les auteurs mais bien des résumés confectionnés par je ne sais qui sans les comprendre suffisamment $\left.{ }^{18} »\right)$ ?

15 Sed nec karpophoros seu carpophorus, de vehiculo invenitur.

16 Tacite, Annales, XII, 43 : uenerationem augebat feminae (" Agrippine rehaussait de plus en plus l'éclat de sa propre grandeur. On la vit entrer au Capitole sur un char suspendu, privilège réservé de tout temps aux prêtres et aux images des dieux, et qui ajoutait au respect du peuple pour une femme de ce rang ").

17 Le terme évoque ici, au sens figuré, le prestige d'Agrippine.

18 SChefFerus, De re vehiculari, p. 420. 
Scheffer omet cependant de dire que Ligorio cite une source numismatique très précieuse pour connaître la forme du carpentum; il s'agit de la médaille frappée par Caligula à l'effigie de sa mère Agrippine décédée, où apparaît son char tiré par deux mulets (fig. 3) et que Scheffer lui-même utilisera dans son chapitre consacré à ce véhicule (fig. 5) ; contrairement à son habitude, Ligorio ne reproduit pas le dessin de la médaille dans la marge de son chapitre ; la raison est sans doute que le dessin de la médaille se trouvait déjà ailleurs dans ses Antichità romane, et plus précisément dans le chapitre consacré aux monnaies d'Agrippine, où la comparaison avec le sesterce original (fig. 4) révèle tout le talent de dessinateur de Ligorio.

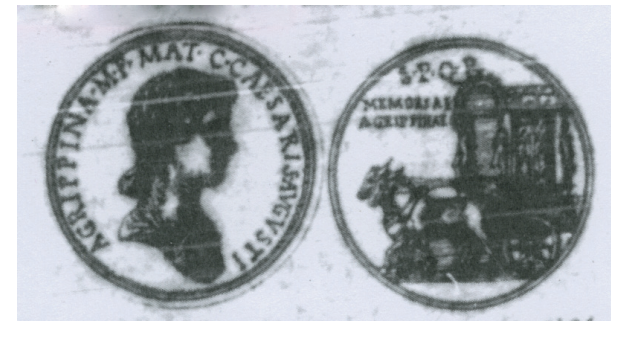

Fig. 3. Torino, archivio di stato, Ja.II.8, f. 127 r.

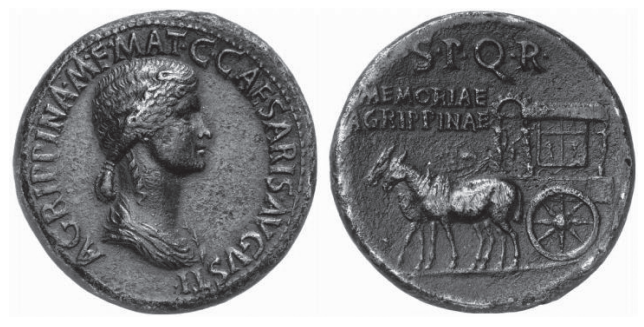

Fig. 4. Sesterce à l'effigie d'Agrippine.

Comme on l'a dit, Scheffer, reproduira à son tour la monnaie d'Agrippine ainsi que celle de Julie (fig. 5), dans le chapitre consacré au carpentum au livre II de son De re vehiculari (II, XVI) ; il s'agit de monnaies qu'il a vues chez Magnus Gabriel de la Gardie (1622-1686), grand chancelier de Suède et favori de la Reine Christine (p. 115 : ex nummo illustriss(imi) Comitis Magni de la Gardie, Regni cancellarij) :
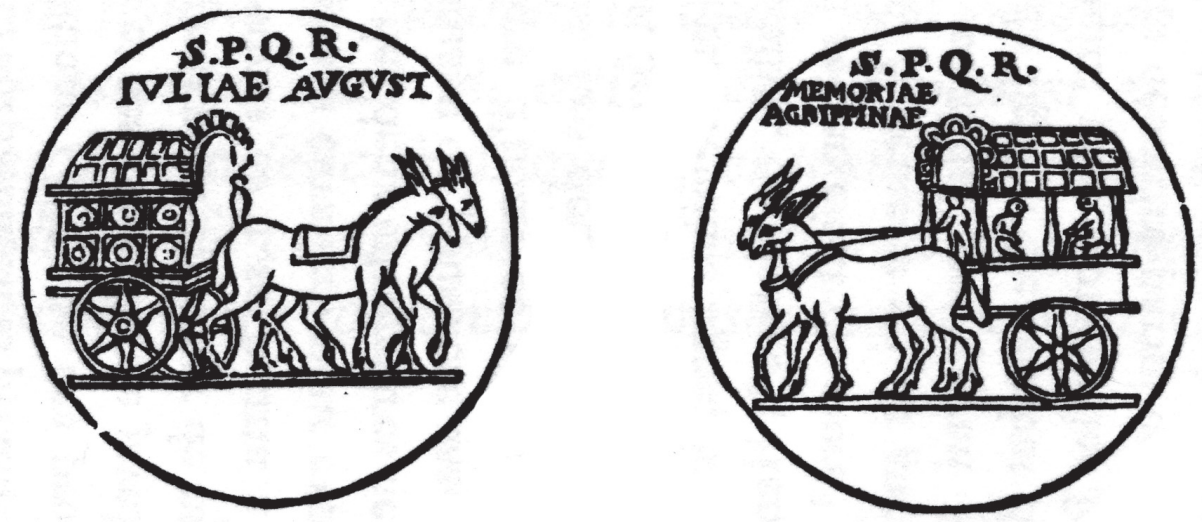

Fig. 5. Carpentum, in J. Scheffer, De re vehiculari, p. 115. 
Pour sa part, Ligorio appellera themsa le véhicule figurant sur la monnaie de Julie, qu'il reproduit dans le chapitre consacré aux monnaies de Tibère (fig. 6), tout en reconnaissant, dans le chapitre de son traité consacré à ce véhicule, qu'il était parfois confondu avec le carpentum ${ }^{19}$ : Non è da dubitar punto in questi rovesci di questa medaglia fatta nel vintesimo quarto anno dellimperio di Tiberio, che questi carri che vi sono non siano il carro chiamato TEMPSA, il quale s'usava menar nelli ludi circensi dopo la morte dell'imperadori, quando erano chiamati divi, il cui honore conseguì Iulia figliola di Augusto che fu moglie di Tiberio et consenso del senato e popolo romano.
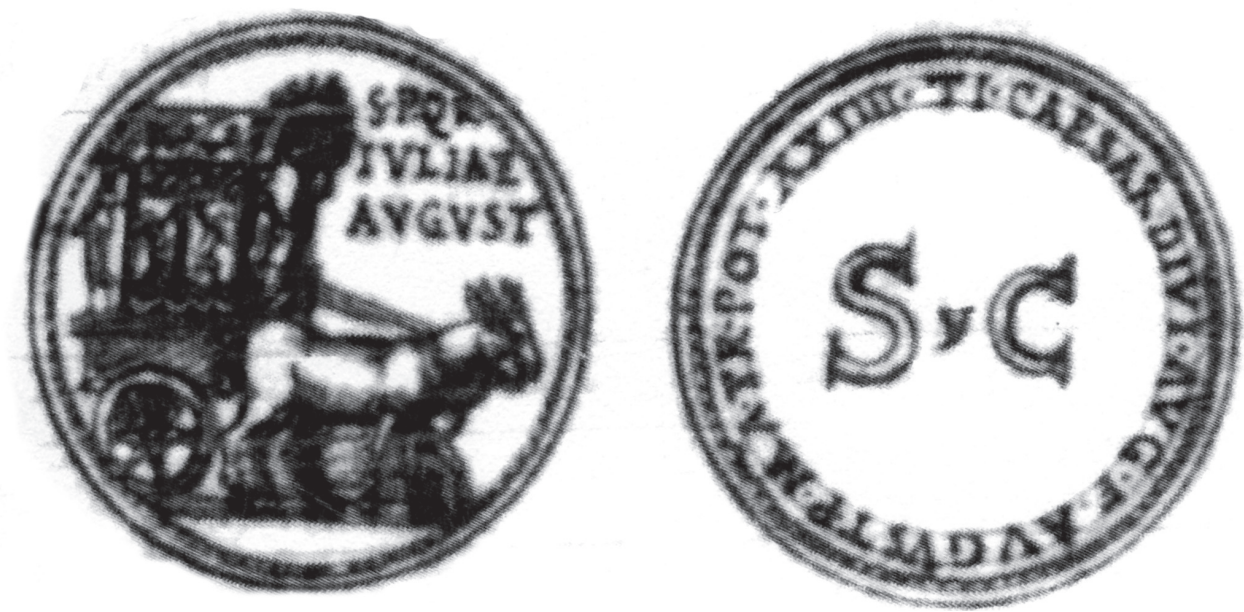

Fig. 6. Torino, archivio di stato, Ja.II.8, f.125r.

À propos du pilentum, dont il est question dans le paragraphe suivant, Ligorio se fonde, une fois de plus, sur les sources littéraires et archéologiques : Pilentum, come se detto, era usato come il carpento detto vehiculo et petorito. Serviva al sudetto officio per portare le matrone colle ceste al sacrificio, lo quale haveva quattro ruote detto in lingua volsca, secondo alcuni; Ma Varrone dice che'l pilento fu trovato in Hispania. Fu usato a tempo suo a Roma, et non prima, dalle matrone nelle cose di sacrificii.

Ici aussi l'antiquaire fait référence à un auteur ancien sans le citer, en l'occurrence Virgile, quand il décrit les matrones qui se rendent aux cérémonies de sacrifices en pilentum, (Énéide, VIII, 865 : castae ducebant sacra per urbem / pilentis matres in mollibus (" de chastes matrones, dans leurs souples chars suspendus, circulaient dans la ville pour accomplir les cérémonies »). Il invoque ensuite, selon son habitude, le témoignage d'un monument antique sur lequel figure le pilentum, la sculpture illustrant la légende

19 Thensa overo thensae sono poste talvolte per carpento et per vehiculo, perché tutti suonano carri, et sacri et privati. 
de Cléobis et Biton, que Ligorio rapporte dans la version de Plutarque ${ }^{20}$ : [...] negli antichi monumenti veggiamo sculpito sifatto carro, con che le donne andavano colle placente a sacrificare a Cerere, colla cesta piena di spiche et di foccacie. Et alcuna fiata hanno due ruote, altri con quatro ruote. Si vede anchora sifatto carro nella casa del Mafei in Roma nell'antica historia di Cleobi e di Bitone argivi, i quali giovanetti invece di buoi tirarono il carro, che alcuni dicono plaustro, altri amaxa, altri vehiculo, altri pilento secondo gli accommoda nel verso la parola, non curando di osservar l'uso del tempo et delle lingue, bastando lor dire un carro dato alla comodità di tali effetti nei sacri misteri usato; col quale andò colle accese facelle la madre di detti giovanetti al tempio di Junone; la quale non potendo havere $i$ buoi attempo, i figlioli tirarono il carro. Et essendo stati gli atti di quelli pietosi et di religiosa gloria, essa sacerdotessa poi pregò la dea per la felicità di quelli ; et tosto amendue s'adormirono nel tempio, né mai più si svegliarono; dal che fu compreso che è meglio la morte nel fine glorioso che vivere con pene e doglie et con pericolo sotto speranza di questa humana vita. Così scrive Plutarco nella consolazione della morte.

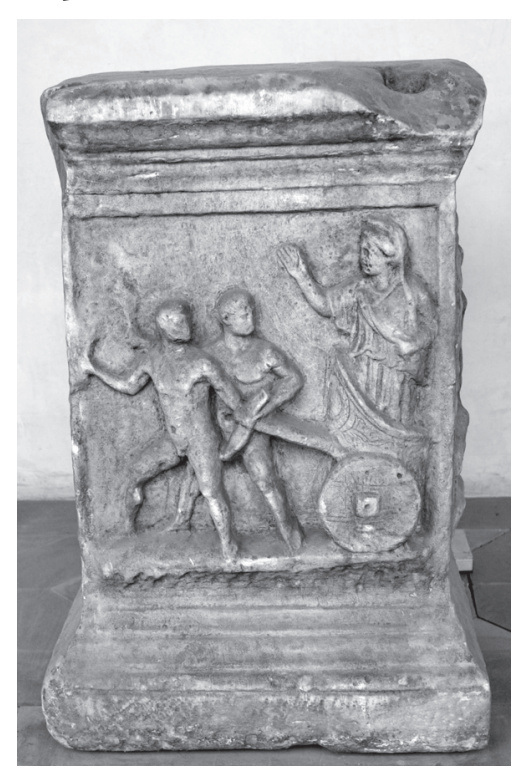

Fig. 7. Rome, Musée des thermes de Dioclétien.

Scheffer, qui reproche à Ligorio de ne pas reproduire les monuments dont il parle, ignorait sans doute l'existence de ce relief conservé jadis dans la collection des Maffei à Rome (fig. 7$)^{21}$.

Comme l'a montré Silvia Tomasi Velli dans son article consacré au cirque romain, un grand nombre de planches publiées en 1600 dans les deux livres De ludis circensibus, sous le nom d'Onofrio Panvinio, avaient été réalisées à partir de dessins de monnaies conservés dans les Antichità romane de Ligorio ${ }^{22}$ : parmi les chars de la pompa circensis (fig. 8), liés aux cérémonies religieuses ou cose sagre, il est vraisemblable que la gravure du char appelé carpenta ait eu comme source le dessin ligorien de la monnaie d'Agrippine.

20 Plutarque, Euvres morales, Consolation à Apollonius, $108 \mathrm{f}$.

21 Utinam nobis eos exhiberet quispiam delineatos diligenter, multum sine dubio iuvarent institutum hoc. ("S'il pouvait nous montrer ces monuments soigneusement dessinés, cela faciliterait grandement notre entreprise. »)

22 S. Tomasi Velli, "Gli antiquari intorno al Circo Romano. Riscoperta di una tipologia monumentale antica ", Annali della Scuola Normale Superiore di Pisa, 1990, p. 148-160 et p. 167-168 ; voir aussi J.-L. Ferrary, Onofrio Panvinio et les antiquités romaines, Collection de l'École française de Rome, 214, Paris-Rome, 1996, p. 33. 


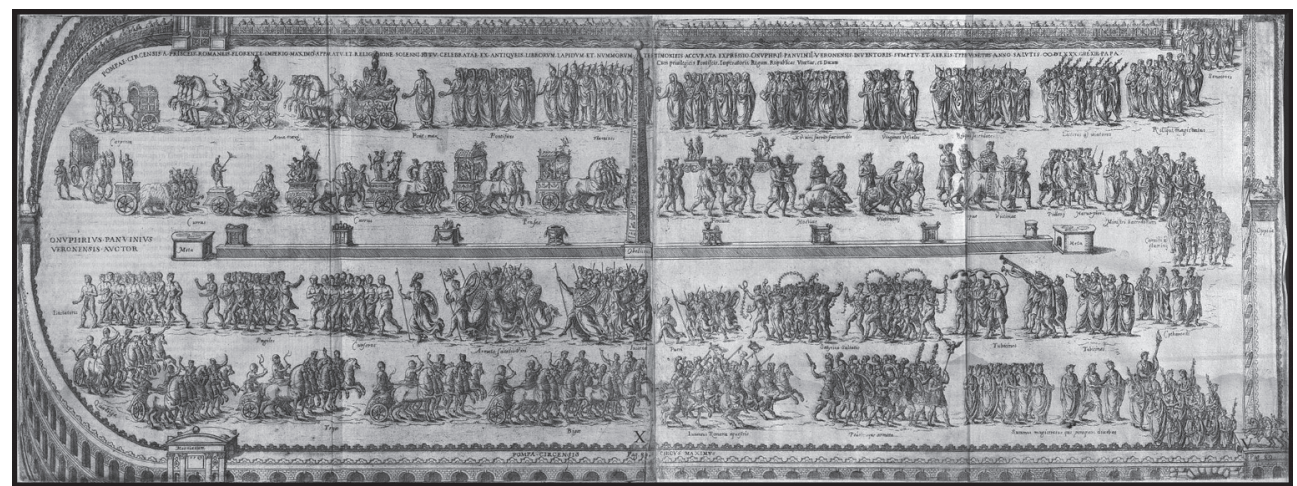

Fig. 8. O. Panviniis, De ludis circensibus, p. 89.

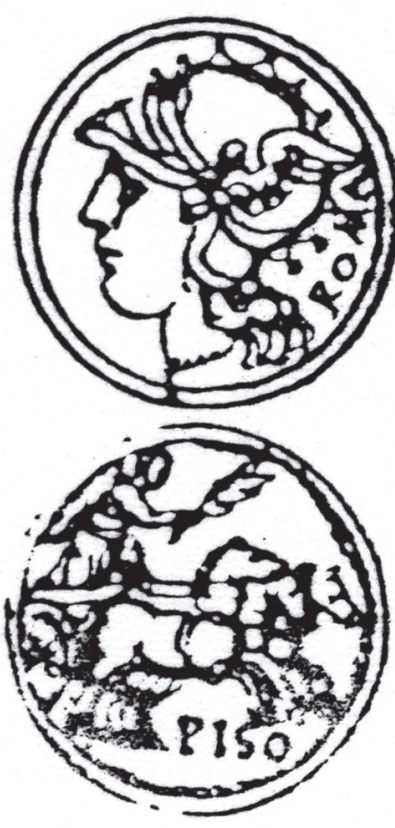

Fig. 9. Torino, Archivio di Stato, Ja.II.6, f.92r.
Quant aux chars de courses représentés sur une autre gravure du livre De ludis circensibus (fig. 14), ils apparaissent à plusieurs reprises dans les Antichità romane et notamment dans le paragraphe consacré aux chars à trois chevaux (DELLA TRIGA CARRO), accompagné cette fois d'un dessin d'une monnaie (fig. 9) : Triga ancora era il medesimo carro, ch'era la biga, detta a triuga, cioè tirata con la congiuntione di tre cavalli al giuogo, come la nominano le antiche memorie scritte in marmo degli agitatori delle fattioni circensi, secondo havemo detto nel libro di giuochi. La qual triga si vede anco nel danaro in questo modo, pure con la vittoria et con altri, che la guidano, come potete veder tra gli altri danari mostrati in quest'opera.

Quand il n'invente pas les monnaies, " de toutes pièces ", comme ce fut probablement le cas pour celle de Caius Alvanius IIIvir, qui trouve sa source dans le texte de Valère-Maxime, Ligorio semble mélanger à dessein plusieurs types de monnaies, à moins que ce soit dû, dans certains cas, à la difficulté d'identifier correctement l'iconographie de deniers républicains ${ }^{23}$; si le nom gravé sous le char (Piso) peut faire penser au

23 Sur la falsification matérielle de monnaies, de la part de Ligorio et d'autres antiquaires, et sur sa conception d'une telle pratique, je renvoie à G. Vagenheim, "Pirro Ligorio et la falsification. À propos du golfe de Santa Eufemia dans la Calabre antique et de la

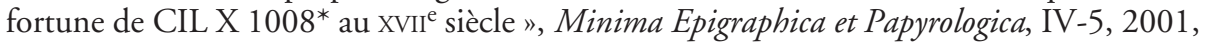
p. 425-449. 
denier de L. Calpurinus Piso dont l'avers montre un cavalier au galop, tenant une palme dans la main ${ }^{24}$, il semble plus juste de voir dans le dessin ligorien une représentation plus ou moins correcte du denier romain républicain d'Appius Claudius Pulcher, émis en 111/110 av. J.-C., qui illustre à la fois un trige et une tête de Rome (fig. 10) ${ }^{25}$. Quant aux memorie scritte auxquelles Ligorio fait allusion, il s'agit de monuments qu'il a reproduits dans ses Antichità romane, soit sur la base de témoignages authentiques comme celui de Cléobis et Biton, soit en les "inventant " de toutes pièces comme le monument à L. Licinus Felix (fig. 11 : CIL VI 2218*).

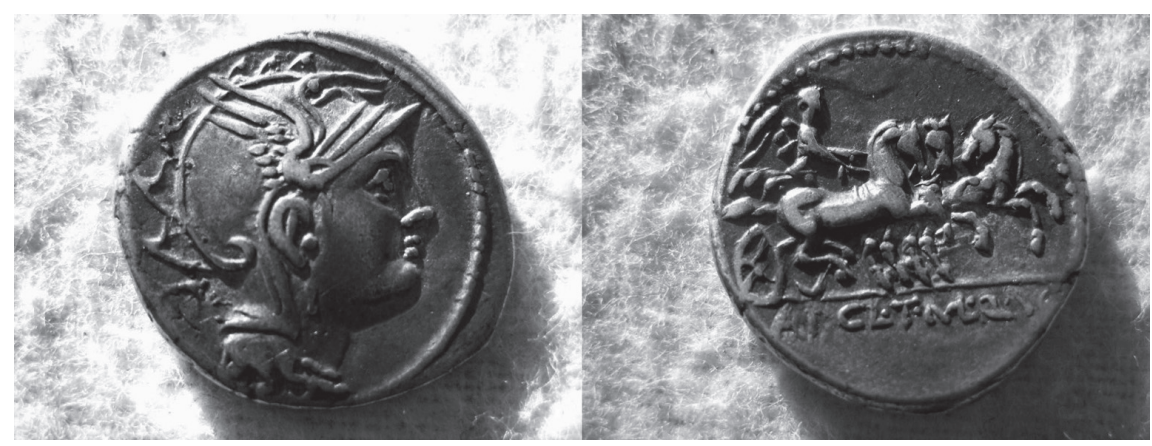

Fig. 10. Denier d'Appius Claudius Pulcher (110-111 av. J.-C.).

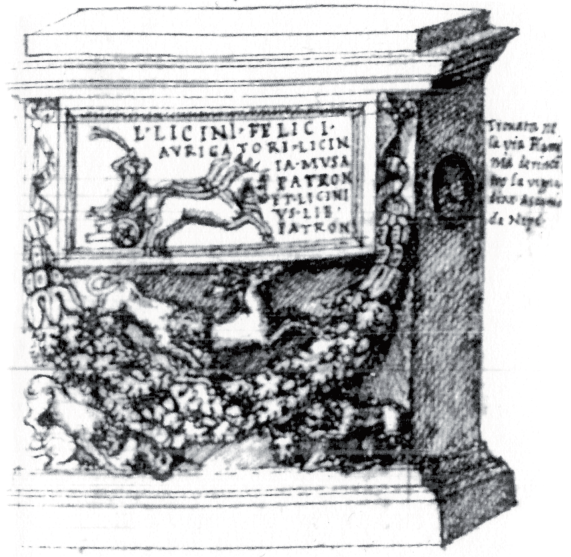

Fig. 11. Napoli, B.N., XIII.B.8, f.92.

24 Voir M.H. Crawford, Roman Republic Coinage, London-New York, Cambridge University Press, 1974, 299/1b ; E.A. Sydenham, The Coinage of the Roman Republic, London, Spik and Son, 1952, 570a.

25 Je remercie François de Callataÿ, directeur d'études en histoire monétaire et financière du monde grec à l'EPHE, pour ces précieuses informations. 


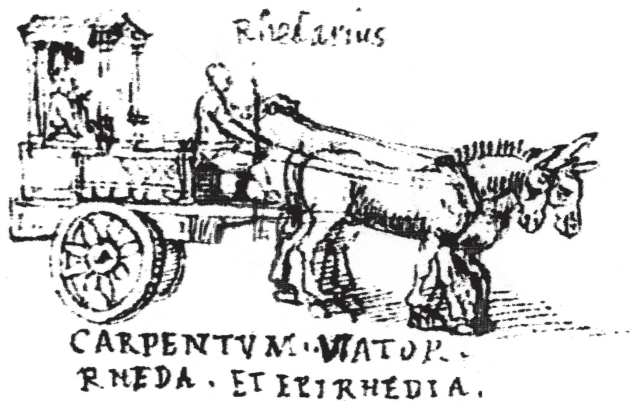

Fig. 12. Stresa, archivio Borromeo.

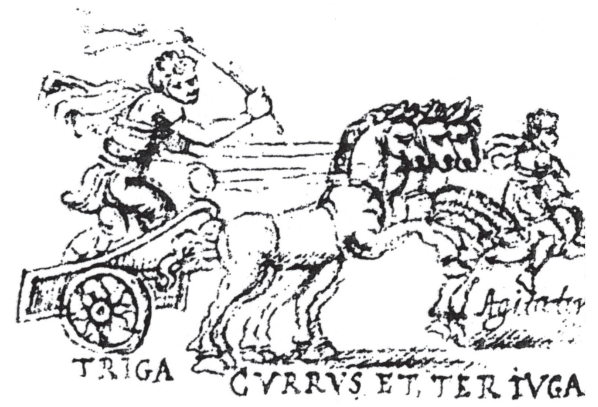

Fig. 13. Stresa, archivio Borromeo.

Finalement, un dessin inédit de Ligorio vient confirmer la distinction qu'il avait établie dans son traité, à propos des véhicules anciens représentés sur les monnaies. Le dessin fut découvert tout à fait par hasard, alors que j'étais sur la trace des dessins de Ligorio qui avaient servi à illustrer le livre du médecin Girolamo Mercuriale sur les exercices de gymnastiques chez les Anciens publié en 1573; une note conservée à la Biblioteca Ariostea de Ferrare, rédigée par le bibliothécaire, le chanoine Giuseppe Antonelli, signalait qu'un membre de la famille Borromée, Giberto, lui avait acheté, en 1856 , les dessins en question appartenant à sa collection privée ${ }^{26}$; j’ai donc eu la chance d'y découvrir les dessins autographes de gymnastique ainsi que d'autres dessins dont un feuillet sur les véhicules anciens ${ }^{27}$.

La partie gauche du feuillet porte des dessins de véhicules liés aux cérémonies religieuses, tel que le carpentum (fig. 12), qui furent ensuite utilisés par les matrones romaines pour se déplacer ; sur la partie droite figurent les dessins des chars utilisés à l'occasion des victoires militaires et qui serviront aussi dans les courses de cirque, comme les triges (fig. 13) ${ }^{28}$.

La rédaction d'un traité ligorien sur les véhicules chez les Anciens s'inscrit parfaitement dans le projet d'encyclopédie universelle des antiquités anciennes, tel que l'avait

26 Pour l'annonce de la découverte, G. Vagenheim, "Some Newly-Discovered Works by Pirro Ligorio ", Journal of the Warburg and Courtauld Institutes, 51, 1988, p. 242-245. La référence à la transaction et la publication d'autres documents inédits conservés dans les archives Borromée se trouvent dans G. Vagenheim, "Lettre inédite de Pirro Ligorio au cardinal Alexandre Farnèse : "Gli abiti delli Iddij chiamati Consenti da Marco Varrone", avec une note de Giovanni Battista Aleotti sur les décors de scène de Pomarancio à Ancône ", in Studi in onore del Kunsthistorisches Institut in Florenz per il suo centenario (1897-1997), Pisa, 1996, p. 236.

27 Je prépare la publication d'épitaphes inédites d'hommes illustres pour les Mélanges offerts à Pierre Laurens.

28 L'ensemble des dessins sera publié dans un prochain article. 
exposé Claudio Tolomei, fondateur de l'Accademia della Virtù, dans sa lettre envoyée à Agostino Landi en $1542^{29}$; c'est finalement Ligorio qui recueillit cet héritage et réalisa ce projet, en rédigeant, avec l'aide des autres membres de l'Accademia degli Sdegnati, ses livres sur les Antichità romane ${ }^{30}$. Le projet de Tolomei mentionnait explicitement la présence d'un livre sur les cirques : [...] i tempii, i portichi, i teatri e gli amfiteatri, le cune, le basiliche, gli archi, le terme, $i$ circi, i ponti, e ogni altro edifizio di che rimanga vestigio alcuno $[\ldots]^{31}$ que Ligorio avait déjà mené à terme au moment où il écrit son traité sur les véhicules anciens (secondo havemo detto nel libro di giuochi) et que Panvinio exploitera, dans une mesure sans doute plus importante qu'on ne l'imagine, pour rédiger et illustrer ses livres De ludis circensibus.

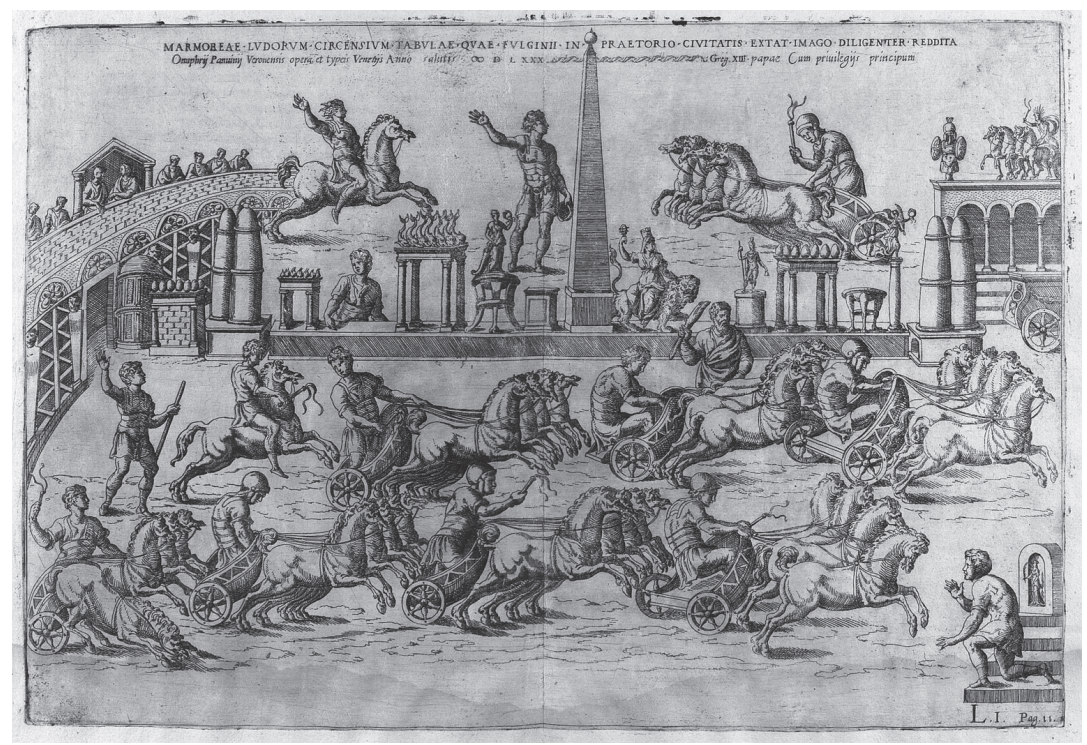

Fig. 14. O. Panvinius, De ludis circensibus, p. 11.

29 Je me permets de renvoyer à G.VAGENHEIM, «Qui a écrit les “Antichità romane” attribuées à Pirro Ligorio (1512-1583) ", in M. Furno., R. MOUREN (dir.), Auteur, traducteur, collaborateur, imprimeur... Qui écrit? Paris, Classiques Garnier, 2012, p. 59-68.

30 Sur l'Accademia della Virtù, on consultera P.N. Pagliara, "Vitruvio da testo a canone ", in S. SetTis (éd.), Memoria dell'antico nell'arte italiana, Torino, 1986, p. 5-85 et sur l'Accademia degli Sdegnati, G.VAGenheim, "Appunti per una prosografia dell'Accademia dello Sdegno a Roma : Pirro Ligorio, Latino Latini, Ottavio Pantagato e altri ", Studi Umanistici Piceni 26 (2006), p. 211-226.

31 Sur la lettre-programme de Tolomei à Landi on consultera M. DALY DAVIS, "Zum Codex Coburgensis : Frühe Archäologie und Humanismus im Kreis des Marcello Cervini ", in R. Harprat, H. Wrede (éd.), Antikenzeichnung und Antikenstudium in Renaissance und Frübbarock. Akten des internationalen symposions 8-10 september 1986 in Coburg, Mainz am Rhein, Philipp von Zabern, 1989, p. 188. 
Pour rédiger son livre sur les cirques, Ligorio a probablement bénéficié de l'érudition de ses amis de l'Accademia degli Sdegnati, et notamment de Benedetto Egio, qui aurait traduit, à son intention, la description du Cirque Maxime de Denis d'Halicarnasse $^{32}$; l'accès des textes anciens à travers des traductions ou des mémoires confectionnés par autrui, comme le suppose Scheffer et comme on le constate dans certains cas $^{33}$, est sans doute l'une des causes des nombreuses interprétations erronées de Ligorio que Scheffer dénonce sans concession et qui révèlent, effectivement, son ignorance des langues classiques ${ }^{34}$. Il n'en reste pas moins que ce même Scheffer avait été fasciné par l'ampleur des connaissances des sources classiques que livrait le traité ligorien sur les véhicules anciens, et qui reflétait l'érudition des humanistes de la dernière académie de la Renaissance consacrée aux antiquités romaines ; ce que n'avait pas mesuré Scheffer, comme on l'a souligné, et qui s'explique par le fait qu'il n'avait de l'œuvre de Ligorio qu'une copie sans dessins, c'est la valeur iconographique inestimable des antichità romane, cette première encyclopédie des antiquités de la Renaissance, qui nous transmet souvent le seul témoignage de monuments aujourd'hui perdus.

La qualité et la précision des dessins ligoriens avaient déjà suscité la convoitise de ses contemporains qui ne manquèrent pas de les utiliser dans leurs publications, comme Panvinio dans les livres De ludis circensibus; il suscitèrent aussi l'intérêt d'autres érudits, comme Orsini qui en tira un album de dessins d'antiquités connu sous le nom de Codex Ursinianus (Vat. lat. 3439); au siècle suivant, Cassiano Dal Pozzo, le mécène de Nicolas Poussin, avait tenté de faire éditer à Paris les Antichità romane; il se contenta finalement d'en tirer le plus grand nombre de dessins, et de les rassembler au sein de son ambitieuse entreprise d'un Museum Chartaceum qui allait servir de répertoire

32 C. Occhipinti, Pirro Ligorio e la storia cristiana di Roma (da Constantino all'Umanesimo), Pisa, Edizioni della Normale, 2007, p. LXXXVI. L'auteur cite un manuscrit inédit conservé à la Biblioteca nazionale centrale de Florence (Autografi Gonnelli, Box 2 C-E) qui porte le titre suivant : Quel che scrisse Dionysio Alycarnasse del Circo Massimo cavato dall'istesso testo greco per me Benedetto Egio fedelmente in questa sentenzia in lingua italiana. Denys d'Halicarnasse, Antiquités romaines, VII, 72, 1-14.

33 Pour des exemples, je renvoie à G. VAGEnheim, "Qui a écrit les "Antichità romane" ", p. 63 et notamment au passage où Ligorio transcrit dans les Antichità romane le commentaire relatif au cassidarius, en renvoyant au livre II de Virgile (nel secondo dell'Eneide) suite à une interprétation erronée du II, qui renvoyait au livre 11 de Virgile dans le commentaire qu'il avait sous les yeux, rédigé vraisemblablement par une tierce personne.

34 Sur la question débattue de la connaissance qu'avait Ligorio des langues anciennes, je me permets de renvoyer à G. VagenheIm, «Le pinceau et la plume : Pirro Ligorio, Benedetto Egio et le Baptistère du Latran ", in M.M. Donato, M. Ferretti (a cura di), Conosco un ottimo storico dell'arte... Per Enrico Castelnuovo. Scritti di allievi e amici pisani, Pisa, Edizioni della Normale, 2012, p. 273-276. 
iconographique pour les artistes, et notamment pour Nicolas Poussin, dont le chevalier Dal Pozzo était le mécène ${ }^{35}$.

Appendice I : J. SCHEFFER, De re vehiculari veterum, Francofurti, ex officina Zunneriana, 1671.

\section{Liber primus}

Cap. I

cap. II

cap. III

cap. IV

cap. V

cap. VI

cap. VII

De vehiculi vocabulo et quid significationis habeat (p. 1)

De vehiculorum primo auctore (p. 7)

De materia vehiculorum (p. 19)

De vehiculorum partibus et temone (p. 29)

De axe (p. 35)

De rotis (p. 42)

cap. VIII

De capso (p. 62)

cap. IX

De animalibus domesticis ad vehicula (p. 79)

cap. X

De feris et similibus domitis ad vehicula (p. 97)

cap. XI

De hominibus ad vehicula iunctis (p. 107)

De jugo et funibus quibus iunguntur (p. 114)

cap. XII

De ratione iungendi (p. 128)

cap. XIII

De armis vectantium seu fraenis (p. 158)

cap. XIV

De flagellis stimulisque (p. 186)

cap. XV

De axungia et similibus (p. 197)

cap. XVI

De vehiculorum ornamentis (p. 203)

cap. XVII

De iis qui quacunque ratione spectant ad vehicula (p. 215)

cap. XVIII

De vario vehiculorum usu (p. 237)

Liber secundus

Cap. I

cap. II

cap. III

De varia et multiplici vehiculorm differentia (p. 1)

cap. IV

cap. V

cap. VI

De clitellis, furculis, aerumnis, et id genus (p. 5)

De ferculis (p. 34)

De sellis cathedrisque (p. 61)

cap. VII

De lecticis (p. 84)

De basternis (p. 115)

De traheis (p. 123)

35 Anthony Blunt avait montré que Poussin s'était inspiré des dessins du Museum Chartaceum pour représenter le triclinium antique dans les scènes de repas des sept sacrements : "The Triclinium in Religious Art ", Journal of the Warburg and Courtauld Institutes, 2 (1938-39), p. 271-276 ; j'ai pu indiquer à mon tour que ces dessins étaient tirés des Antichità romane de Ligorio: G.VAGENHEIM, "Des inscriptions ligoriennes dans le "Museo Cartaceo". Pour une étude de la tradition des dessins d'après l'antique ", in Cassiano Dal Pozzo's Paper Museum, I, 1992, p. 79-104. 


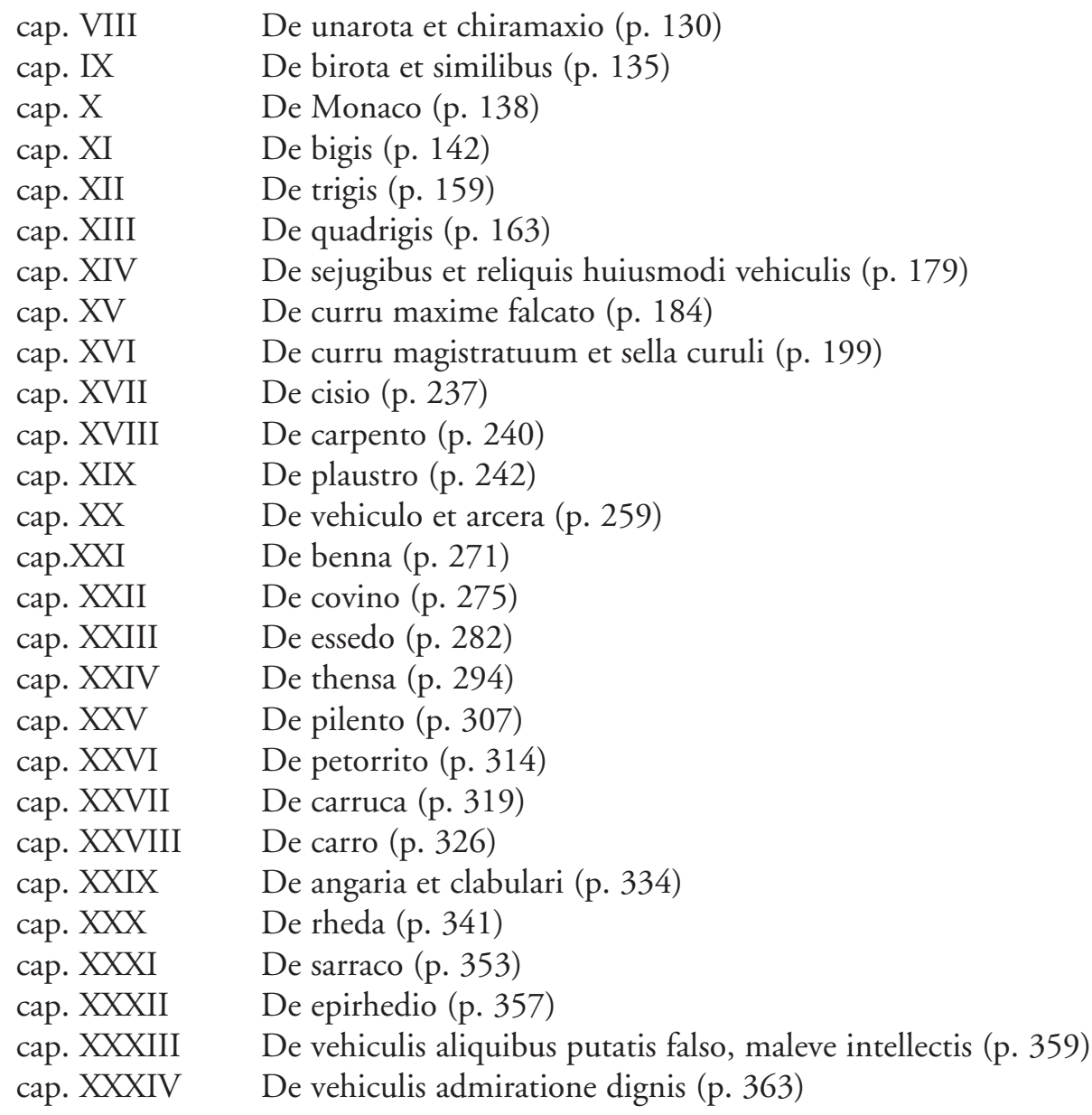

Appendice II. P. Ligorio, Dell'Alvanii et della sorte de carri antichi, Torino, Archivio di Stato, Ja.II.6 ff.89r-94v.
I. Del plaustro.
II. Del carpento.
III. Del vehiculo, pilento e petorito.
IV. De vehiculo detto cisio et carruca.
V. Della carruca et rheda.
VI. Della thensa et carpento et vehiculo.
VII. Del curro biroto et deipaneta et vehiculo.
VIII. Del burdones vehiculo.
IX. Del camulon vehiculo.
X. Del cantherio vehiculo. 
XI. Del combatus vehiculus et comba.

XII. Del covinus vehiculus.

XIII. Currus vehiculus et curritius.

XIV. Curro et esseda.

XV. Di arcera, arcirna et plaustrum.

XVI. Basterna et vehiculo.

XVII. Benna et vehiculo.

XVIII. Biga carro overo curro biuga.

XIX. Della triga carro.

XX. Della triga et quadriga pilento et curro et quadriga.

XXI. Del veha, carro rustico.

XXII. Del octophoro vehiculo et lectica.

XXIII. Del carro detto petorito.

XXIV. Del plaustro vehiculo di Marte detto plostro.

XXV. Rheda, vehiculo et epirhedia.

XXVI. Del sarraco vehiculo.

XXVII. Del staticulo vehiculo.

XXVIII. Della traha et tribula.

XXIX. Del vectabulum.

Ginette VaGenheIm

ERIAC (Université de Rouen)/IUF
Université de Haute-Normandie
F-76821 Mont-Saint-Aignan Cedex
ginette.vagenheim@univ-rouen.fr

